\title{
Study of secondary muons detected within the tunnels of the Cholula pyramid
}

\author{
E. MORENO-BARBOSA ${ }^{1 *}$, J.E. ESPINOSA-ROSALES ${ }^{1}$, J. COTZOMI-PALETA ${ }^{1}$, \\ B DE CELIS-ALONSO ${ }^{1}$, M. CRUZ-SÁNCHEZ ${ }^{2}$, E. MERLO-JUÁREZ², P. TREJO- \\ GARCÍA $^{1}$, A. LIMA-FLORES ${ }^{1}$, R. PALOMINO-MERINO ${ }^{1}$
}

${ }^{1}$ Facultad de Ciencias Físico Matemáticas, Benemérita Universidad Autónoma de Puebla, Avenida San Claudio y 18 Sur, Col. San Manuel, Ciudad Universitaria, Puebla, Puebla. C.P. 72570, México.

${ }^{2}$ Instituto Nacional de Antropología e Historia , Delegación Puebla, Zona Arqueológica de Cholula, 8 norte núm. 2, colonia centro, San Andrés Cholula, Puebla, C.P. 72760 , México.

\section{*Email: emoreno@fcfm.buap.mx}

Published online: August 07, 2017

The Author(s) 2017. This article is published with open access at www.chitkara.edu. in/publications

\begin{abstract}
The pyramid of Cholula was built at the beginning of 100 B.C. and during of period of 500 years it was finished, had several new constructions, based on the previous constructions. The primarily material of construction is the adobe. Early in 1931 archaeological excavations began with the intention of exploring the interior of the pyramid, excavations were stopped in 1971, and to date no further excavations have been carried out. This work shows the first measurements of muons, particles that are very penetrating, these are generated by primary cosmic rays that was incoming in the atmosphere and these generates a rain of secondary particles, among them the muons. To measure this kind of particles was implemented a detector system, it is formed by a scintillator plastic coupled to a tube photomultiplier; the signals were acquired by mean of an oscilloscope. The detector was collocated near of the center of the pyramid; the location belongs to the maxima concentration in mass over the detector. Graphs of the charge distribution, maximum amplitude and characteristic rise times of the generated pulses in a plastic scintillator are shown, this is scintillator was synthesized in the materials laboratory of the FCFM-BUAP. In addition the optical characterization of the same was realized.
\end{abstract}

Keywords: Scintillation detector, Cosmic rays, Tube photomultiplier, Pyramid
Journal of Nuclear

Physics, Material

Sciences, Radiation and

Applications

Vol-5 No-1

August 2017 pp. $45-52$ 
Moreno-Barbosa, E.

Espinosa-Rosales, J.E. Cotzomi-Paleta, J.

Celis-Alonso, B De

Cruz-Sánchez, M.

Merlo-Juárez, E.

Trejo-García, P.

Lima-Flores, A.

Palomino-Merino R.

\section{INTRODUCTION}

High-energy cosmic rays hit constantly the earth's atmosphere and interact with the air molecules; these are characterized by the measurements of the generated secondary particles. These bunches of particles are called extensive air showers (EAS). Eventually these could reach the surface of the earth. The shower has two principal components, the muonic and the electromagnetic (electrons, positrons and photons). Muons are mainly produced from the decay of charged mesons and have a decay length of $15 \mathrm{~km}$, this mean that are produced high in the atmosphere and they are very penetrating. This feature of penetration allows detecting the muons, when they pass through a great amount of matter like rock. The principal objective of this work is quantify the temporary structure of muon signal inside of the pyramid of Cholula (Figure 1 left), by mean of homemade scintillator plastic that was placed near at the center of it basis and where is the largest amount of building material on it (approx. 65 meters), which is mainly adobe (clay and sand mixed with straw) some sections around was covered with stone. The scintillator plastic has been used in diverse experiments of high energies like: Pierre Auger (AMIGA, BATATA detectors), LHASOO, EAS- TOP, some used as discriminators and other used for the energy and arrive direction of EAS [1,2]. Another applications of the scintillator plastic or drift chambers and the natural flux of muon spectrum in others areas that are not particles physics or astroparticles, are the generation of imaging (radiography or tomography) using muon
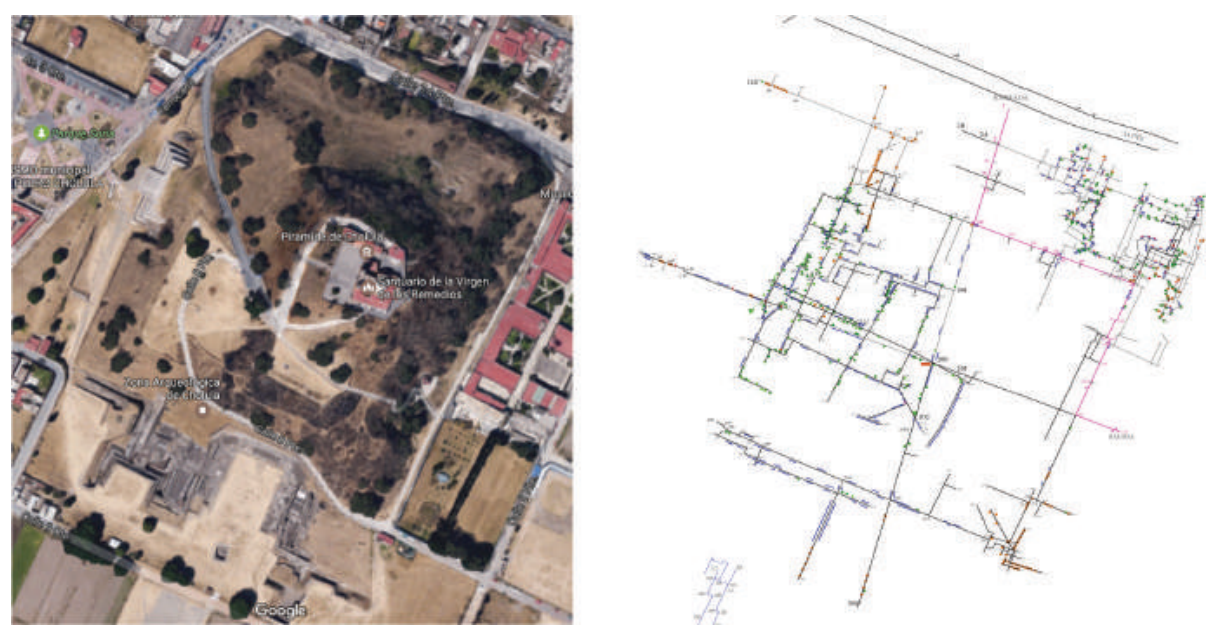

Figure 1: (Left) Top view of extension of the archeological zone and the pyramid (picture from Google maps), (right) Map of the inner tunnels of the pyramid, the orange rectangle shows the place where the detector system was placed. 
attenuation has been used since the mid-1950s to study mines [3], caverns [4], pyramids [5], volcanoes [6], and nuclear reactors. [7,8]. It is possible make an approximation of the muon attenuation given by

$$
-\frac{N}{\lambda}=\frac{d N}{d x}=\frac{d N}{d E} \frac{d E}{d x}
$$

$\mathrm{N}$ is the number of muons, lambda is the attenuation length, $\mathrm{dN} / \mathrm{de}$ is the muon energy spectrum, $-\mathrm{dE} / \mathrm{dx}$ the mean energy loss rate.

\section{MATERIALS AND METHODS}

\subsection{Detector description}

The detector media for the charged particle is a plastic scintillator material homemade (figure 2 left) [9]. It is a circular piece of $20 \mathrm{~cm}$ of diameter and $5 \mathrm{~cm}$ of thick, was made with Polystyrene (C8H8) and mixed with two flours: POP (2,5-diphenyloxazole) and POPOP (1,4-bis-2-5-phenyloxazoly) benzene with a proportion of $1 \%$ and $0.03 \%$ respectively, before the plastic was extruded in a circular recipient. A face of the plastic was placed in contact with a photomultiplier tube (PMT) of $12 \mathrm{~cm}$ of diameter. The surface of the plastic that are not in contact with the PMT was covered with Tyvek®, which is a material with a highly reflectivity and diffuser, a second coating was performed with black tape for prevent light input from outside.

The input analog signals from the PMT are acquired by means of a digital oscilloscope controlled by the LabVIEW software for signal acquiring.

The signals were post-processed using a program developed in LabVIEW for the calculation of four characteristic parameters: charge deposited,
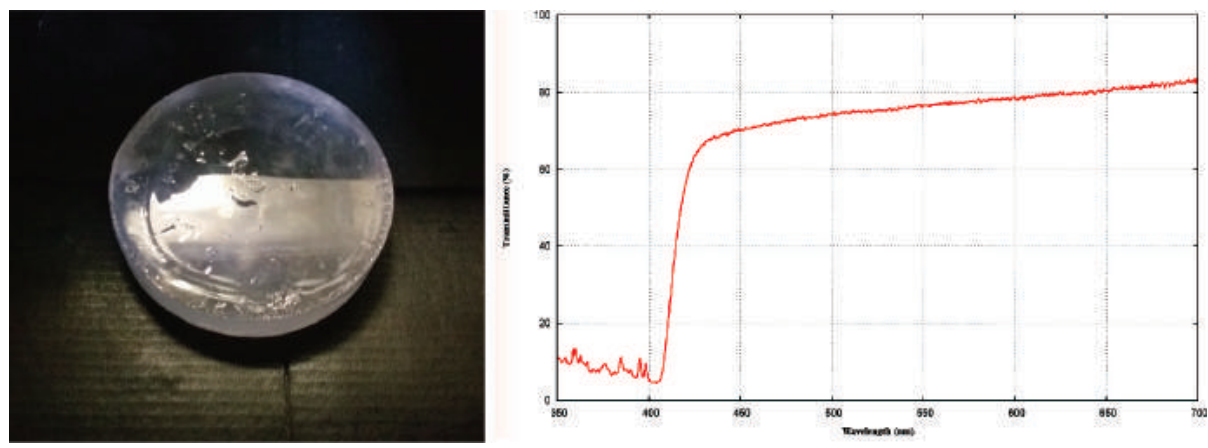

Figure 2: (Left) Scintillator plastic development at the materials laboratory of the FCFM BUAP, (right) Transmission curve of the scintillator, for the maximum of the spectral response of the PMT used, the scintillator shows a $30 \%$ of attenuation.
Study of secondary muons detected within the tunnels of the Cholula pyramid 
Moreno-Barbosa, E. maximum voltage, and two signals related with the duration or widths of the Espinosa-Rosales, J.E. signal, t50 and t90 they are the time interval in which the integrated charge Cotzomi-Paleta, J.

Celis-Alonso, B De

Cruz-Sánchez, M.

Merlo-Juárez, E.

Trejo-García, P.

Lima-Flores, A.

Palomino-Merino R. of the individual signals rise from $10 \%$ to $50 \%$ and $90 \%$. These parameters are distributed differently depending on the type and energy of particle that interacts with the scintillator [10-13]. All data were analyzed using the ROOT program, a data analysis framework [14].

\subsection{Setup assessment measurements}

The system was placed in near center at the base level of the pyramid, the figure 1 (right) shows the position where was placed the system in the archaeological tunnels of the pyramid. In that point above the detector system is the maximum amount of building material and therefore it is expected to have the highest particle attenuation. For the particle detection a constant voltage of -1400 volts of polarization was applied to the photomultiplier tube, in a previous test it was found that the plate region for this detector is greater than $-1300 \mathrm{~V}$. The input analog signals from the PMT are acquired by means of a digital oscilloscope (Tektronix TDS220 @ 200Mhz BW), which is controlled by the LabVIEW software for signal acquiring. A discriminator threshold (Vth) for the acquisition of the signals from PMT, was increasing in steps of $2 \mathrm{mv}$ from 60 to $150 \mathrm{mv}, 20000$ samples were taken for each Vth.

\section{RESULTS}

The figure 3 shows the histograms of four parameters of a threshold voltage of $6 \mathrm{mv}$ in the interior of pyramid. Histogram of a charge deposited (top left)
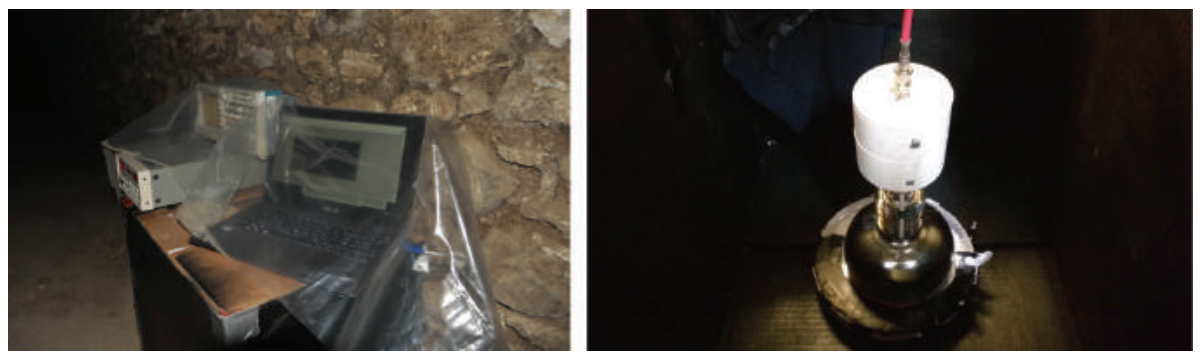

Figure 1: The acquisition system was placed approximately in the center of pyramid, around 200 meters of the principal entrance and 200 of the exit of the touristic tour. In the right picture shows the system detector where it was put, a commercial oscilloscope acquire de signal from the PMT and the laptop control and save the signal observed. The plastic scintillator coupled with a tube photomultiplier inside of black box for prevent humidity and light. 

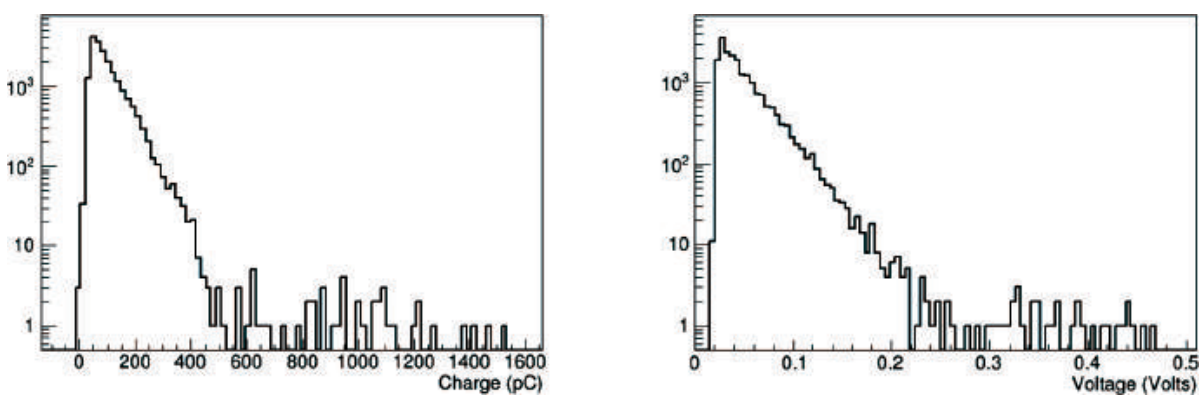

Study of secondary muons detected within the tunnels of the Cholula pyramid
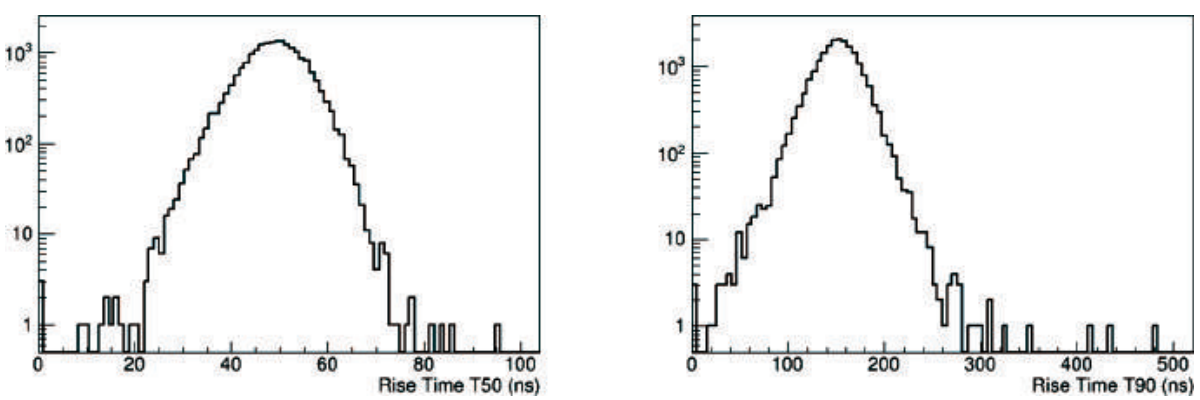

Figure 3: Histograms of Charge (pC), Voltage (mv) and rise time t50 and t90 when a threshold voltage of $6 \mathrm{mv}$ was setup.

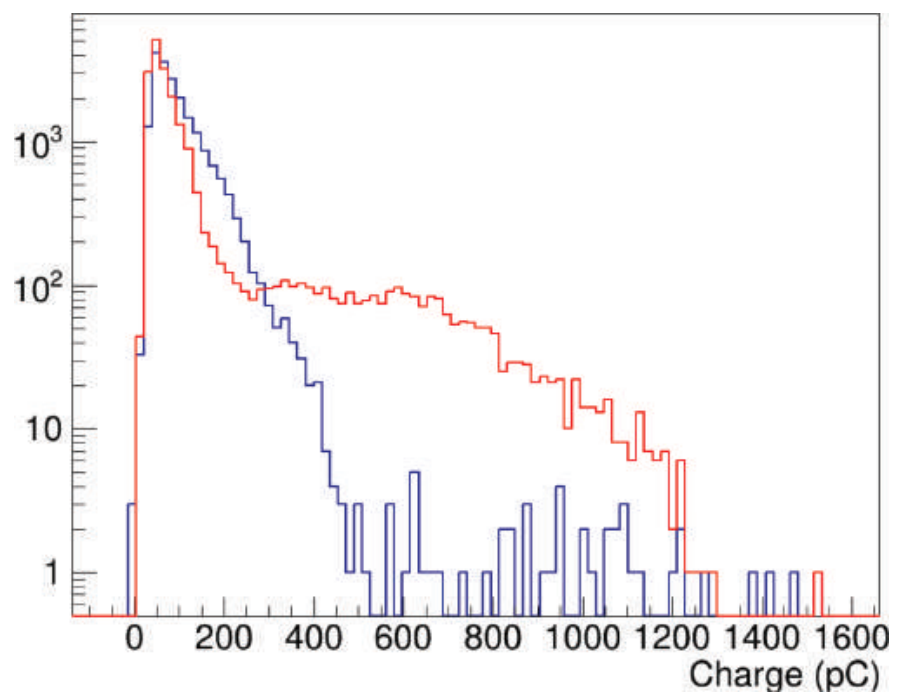

Figure 4: Histograms of charge, the blue line correspond to the data in the pyramid and the red line corresponds to data acquired in the laboratory with a $6 \mathrm{mv}$ of Vth. 
Moreno-Barbosa, E. in the medium by the particle, the maximum voltage of the signals (top right) Espinosa-Rosales, J.E. and the time rise t50 (bottom left) and t90 (bottom right). With the same Cotzomi-Paleta, J.

Celis-Alonso, B De Cruz-Sánchez, M. Merlo-Juárez, E. Trejo-García, P. Lima-Flores, A. conditions of polarization in the tube photomultiplier and whit $6 \mathrm{mv}$ of Vth for the trigger was take the equal sample of signal on the laboratory in the campus of university, the figure 4 show both charge distributions. A two-dimensional correlation of charge versus T50 is show in the figure 6, in the top of the graph it is possible observe a few events with high charge and T50 .

Palomino-Merino R.

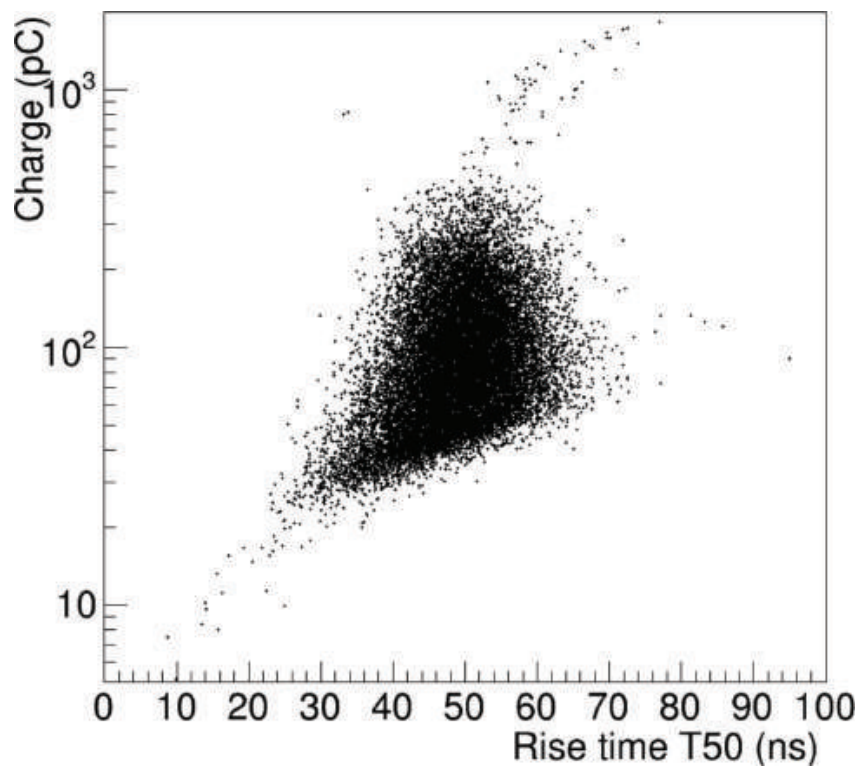

Figure 6: Correlation between the Charge and rise time of 50 percent of all particles detected, with the lowest threshold voltage for the acquired signals $(6 \mathrm{mv})$.
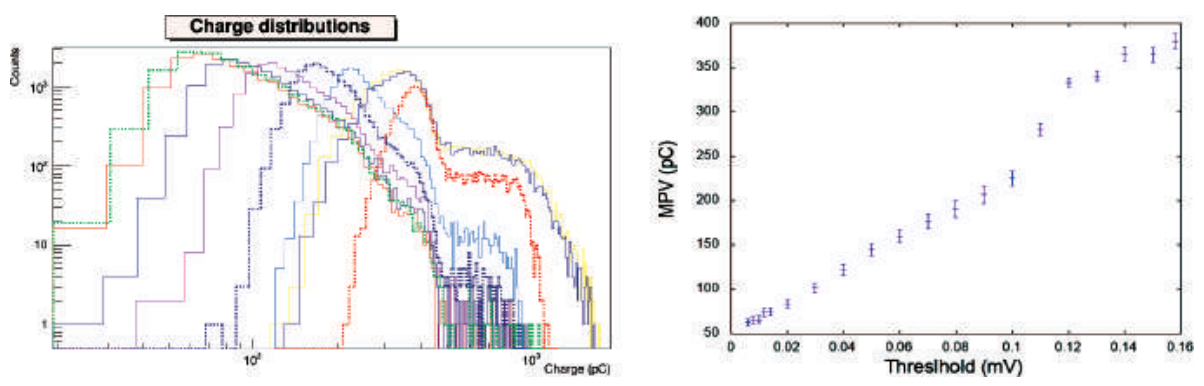

Figure 7: (Left) Histograms of charge deposited in the scintillator plastic. (Right) Plot of the most probably values against the variation of the voltage threshold of the trigger for each histogram of charge taken. 
Characteristic charge histograms and the dependence of the most probable value (MPV) versus reference voltage are show in figure 7. In each histogram of charge obtained from the variation of the Voltage threshold, was used a landau function in order to fit the shape and obtain the MPV.
Study of secondary muons detected within the tunnels of the Cholula pyramid

\section{DISCUSSION AND CONCLUSIONS}

The histogram of charge inside of pyramid (Figure 3 top-left) shows a decrease of values when the integrated charge is greater than $600 \mathrm{pC}$, this is due to that pyramid has function of filter and the particles of the extensive air shower do not reach the scintillator. The dispersion graph of charge versus time of rise (T50) has a behavior similar to that reported in the literature when is analyzing the signals in a detectors of the kind water Cherenkov detector, this type of two-dimensional representation can help to discriminate between different types of particles and may even could be used for calibration of the detector. As conclusion was synthesized plastic optimizing the dopants for scintillating, the scintillator at the passage of particles, have the capacity to detect furthermore, it is possible to observe temporal structure even with a large amount of material.

\section{ACKNOWLEDGEMENTS}

We want to thank to Instituto Nacional de Antropología e Historia - Puebla for allowing us the access to the inside the pyramid. For all help and support also for the historical background.

\section{REFERENCES}

[1] R. Alfaro et al., (2010) Buried plastic scintillator muon telescope (BATATA), Nuclear Instruments and Methods in Physics Research A 617, 511-514.

[2] A. Aab et al., The Pierre Auger Observatory Upgrade - Preliminary Design Report, arXiv:1604.03637v1 [ASTRO-PH.IM], 13 Apr 2016.

[3] E.P. George, (1955) Cosmic rays measure overburden of tunnel, Commonwealth Engineer, 455-457.

[4] L.W. Alvarez et al., (1979) Search for hidden chambers in the pyramids, Science, 167(3919), 832-839.

[5] H. K.M. Tanaka, (2008) Radiography imaging below a volcanic crater floor with cosmic-ray muons, American Journal of Science, 308, 843-850.

[6] E. Caffau, F. Coren, and G. Giannini, (1997) Underground cosmic-ray measurement for morphological reconstruction of the "Grotta Gigante" natural cave, Nuclear Instruments and Methods in Physics Research A, 385(3), 480-488. 
Moreno-Barbosa, E. [7] H. Miyadera et al., Imaging of a reactor with mouns, Nuclear Science Symposium Espinosa-Rosales, J.E. Cotzomi-Paleta, J. Celis-Alonso, B De Cruz-Sánchez, M. Merlo-Juárez, E. Trejo-García, P. Lima-Flores, A. and Medical Imaging Conference, IEEE, (2013)

[8] J. Perry et al., Imaging a nuclear reactor using cosmic ray muons, Journal of Applied Physics, 113, 184909 (2013)

[9] E. Moreno-Barbosa et al. (2016) Proposal of a novel Setup for LINAC monitoring using a specifically designed plastic scintillator and a spectrophotometer, Journal of Nuclear Physics, Material Sciences, Radiation and Applications 4(1), 175-182. Palomino-Merino R.

[10] H. Salazar et al. (2003) Signal Temporal profile of a water Cherenkov detector., AIP Conference Proceedings, 674(381), 1-4

[11] J. Cotzomi et al. (2005) The water Cherenkov detector array for studies of cosmic rays at the University of Puebla., Nuclear Instruments \& Methods in Physics Research A, 553(1), 290-294.

[12] J. Cotzomi et al. High altitude water Cherenkov Detector performance., ICRC 2011, (2011)

[13] A. Galindo et al., (2017) Calibration of a large water-Cherenkov detector at the Sierra Negra site of LAGO., Nuclear Instruments and Methods in Physics Research Section A 861, 28-37.

[14] Root Analysis Framework, http://root.cern.ch 